\section{Toxicología clínica comunitaria}

\section{Community Clinical Toxicology}

M aría Elena Leal ${ }^{1}$

Diego González ${ }^{2}$

Susana I sabel García ${ }^{3}$

I ván N elinho Pérez-M aldonado ${ }^{1}$

Edna Rico ${ }^{4}$

Patricia Cossío ${ }^{1}$

Evelyn Van Brussel ${ }^{1}$

Gabriela Domínguez ${ }^{1}$

Fernando Díaz-Barriga ${ }^{1}$
${ }^{1}$ Departamento de ToxicologíaAmbiental, Facultad deM edicina, Universidad Autónoma de San Luis Potosí, Centro ColaborantedelaOMS/ OPS en Evaluación de Riesgos y Salud Ambiental Infantil. Avenida Venustiano Carranza 2405, Col Lomas los Filtros. 78210 San Luis Potosi SLP M éxico.

fdia@uaslp.mx

${ }^{2}$ Organización

Panamericana dela Salud.

${ }^{3}$ M inisterio de Salud, Argentina.

${ }^{4}$ Centro de Estudios

Básicos, Universidad

Autónoma deCiudad Juárez.
Abstract In some Latin A merican countriesacute intoxication is professionally managed by specialized physicians qualified in the area. Something similar occurs with work-related chronic intoxication in the formal sector. However, a different reality prevails for the assessment of chronic intoxication of environmental origin, since it is by definition more difficult to diagnose. For treatment of acute intoxication, Toxicological Information and $C$ are Centers have been set up, though similar bodies have not been created for chronic environmental intoxication. Therefore, in this study a model of chronic intoxication care is proposed, using multidisciplinary teams adopting a new approach, namely Community Clinical Toxicology, the goal of which would be the simultaneous care of acute intoxication which is generally treated in hospital, and environmental intoxication that is normal dealt with at community level. Theultimate goal is to take advantage of the expertisethat existsin the Region in terms of Clinical Toxicology to organize community work.

Key words Toxicology, Clinical Toxicology, Community, Contamination, Environmental Health
Resumen En algunos países de América Latina las intoxicaciones agudas se manejan de manera profesional por médicos especialistas en la materia. Algo similar ocurre con las intoxicaciones crónicas de origen laboral en el sector formal. N 0 obstante, una realidad diferente ocurreen cuanto a la evaluación de las intoxicaciones crónicas de origen ambiental, dado que éstas por su naturaleza, son más difíciles de diagnosticar. Para el tratamiento de las intoxicaciones agudas se han organizado Centros de Información y Atención Toxicológica, pero para las intoxicaciones crónicas ambientales no seha generado organismossemejantes. Por consiguiente, en este trabajo sugerimos un modelo de atención de la intoxicaciones crónicas a través de grupos multidisciplinarios bajo el esquema de una nueva disciplina: Ia Toxicología Clínica Comunitaria, cuyo objetivo sería la atención simultánea de las intoxicaciones agudas que generalmente se atienden en un ámbito hospitalario y de las intoxicaciones ambientales que por lo normal se presentan a nivel comunitario. El objetivo final es aprovechar la experiencia que existe en la Región en cuanto a Toxicología Clínica para organizar el trabajo comunitario.

Palabras clave Toxicología, Toxicología Clínica, Comunidad, Contaminación, Salud Ambiental 
El esquema

La exposición crónica a contaminantes puede presentarse en las grandes metrópolis como resultado de las emisiones proveniente de miles de automotores. Pero también puede darse en sitios contaminados (por residuos industriales, por agroquímicos, etc.); y/o en comunidades marginadas (por el uso de biomasa al interior de la vivienda, ingesta de agua contaminada, empleo de barro vidriado con plomo, etc.); y/o en áreas impactadas por emergencias naturales (inundaciones que desbordan cuerpos de agua contaminados, incendios forestales, etc.).

Además, la exposición ocupacional a químicos, sobre todo en el sector informal, va en incremento en la medida que van aumentando los individuos que laboran en condiciones poco favorables para la salud (minería artesanal, fabricación de ladrillo, carpintería, reciclado de basuramunicipal, etc.).

Aunado a lo anterior, en estos sitios donde ocurrela exposición crónica, otros hechos merecen nuestra atención, ellos son: las exposiciones acumuladas y las exposiciones agregadas. Las primeras implican contacto simultáneo con varios tóxicosque presentan entresí similares efectos tóxicos (por ejemplo en zonas mineras se puede dar la exposición a metales neurotóxicos, en tanto en campos agrícolas se puede dar la exposición a varios insecticidas inhibidores de la colinesterasa). Por su parte, exposiciones agregadas son aquellas que se dan cuando ocurre contacto con un mismo contaminante pero por múltiples rutas, así por ejemplo, en zonas maláricas dónde hubo aplicación de DDT, puede haber contacto con esteinsecticida persistentea través de la ingesta de suelo/polvo, de la ingesta de alimento (peces o leche materna) y de la inhalación de vapores (al volatilizar el compuesto residual todavía presente en el suelo).

Las exposiciones acumuladas y/o agregadas, pueden dar lugar a escenarios mas complejos, tal es el caso de las áreas mineras del Amazonas, dondeademás del mercurio puede haber exposición a plaguicidas empleados para el combate de la malaria o el dengue; o el caso de las comunidades indígenas que al quemar leña en interiores permiten la exposición a tóxicos tan diversos como hidrocarburos aromáticos policíclicos, monóxido de carbono, formaldehídos, etc.; por cierto, estas comunidades en muchas ocasiones también presentan exposición a microorganismos patógenos, sobre todo en el agua de consumo. En estos y otros escenarios, debe contemplarse que los efectos crónicos pueden exacer- barse por desnutrición o al presentarse la exposición en etapas de extrema vulnerabilidad como en las etapas fetales o infantiles. En estas etapas los efectos pueden aparecer a menores dosis que las requeridas para los adultos ${ }^{1}$.

En conclusión, múltiples son los escenarios donde ocurre la exposición crónica a sustancias tóxicas y por consiguiente, el número de individuos afectados es grande. Por eso llama la atención que la atención médica delasintoxicaciones crónicas sea tan limitada. Una exposición crónica a bajas dosis, a pesar de que finalmente puede manifestarse en síntomas y signos de enfermedad, con dificultad cuenta con la atención del personal médico, sobre todo debido a la dificultad del diagnóstico y al costo de los estudios que son requeridos para establecer una conexión entre la exposición y el efecto. Además, a diferencia de las intoxicaciones agudas que son consultadas a los CIAATs (Centros de Información, Asesoramiento y Asistencia Toxicológica), para las exposiciones crónicas especialmente las de origen ambiental, no se cuenta con un sistema de atención toxicológica organizada.

¿Cómo responder entonces al reto de la atención a las intoxicaciones crónicas ambientales en las comunidades expuestas?

La atención dela exposición crónica requiere de un modelo de trabajo y para ello, hemos considerado útil la unión de dos disciplinas: la toxicología y la evaluación de riesgos. La primera se ha desarrollado desde épocas antiguas validando los efectos nocivos de sustancias que afectan al hombre. A lo largo del desarrollo de la Toxicología como disciplina, se han logrado conceptos tan sólidos como el que "todas las sustancias son venenosas a la concentración adecuada". La toxicología habla de agresiones y de resistencias a nivel celular y cuando los mecanismos de daño sobrepasan a los mecanismos de protección, se manifiesta al gún efecto biológico que puede desembocar en enfermedad. Sin embargo, ahora los nuevos conocimientoshan desembocado en nue vos conceptos y entre otros, hoy en día se sabe que "la concentración necesaria para que una sustancia sea tóxica depende del instante en el desarrollo del ser vivo durante el cual ocurre la exposición al veneno"1 ${ }^{1}$. En ciertos momentos de la vida, las células son mas susceptibles al ataque de elementos tóxicos, tal es el caso de las fases embrionaria y fetal del desarrollo; la exposición durante estas "ventanas de vulnerabilidad" pue de dejar una huella que puede manifestarse de inmediato, por ejemplo en forma de una malformación congénita, o puede manifestarse años después del nacimiento en algún otro padecimien- 
to como alteraciones neurocognitivas, endócrinas o cáncer ${ }^{1}$. Así que ahora no importa solamenteel "cuánto" (concentración requerida) sino también el "cuándo" ocurre la exposición; y aquí ligamos la toxicología con la evaluación de riesgos, ya que precisamente el método de evaluación de riesgos nos permite definir parámetros que nos llevan a establecer el "cuándo" ocurre la exposición y además nos aporta datos para establecer cual es la población afectada y por cuales rutas se está exponiendo.

Así que por un lado contamos con la toxicología y sobretodo con su especialidad clínica para la evaluación del ser humano; en tanto por otro lado, contamos con la evaluación de riesgos que a nivel comunitario nos facilita establecer magnitudes y temporalidades dela exposición. Al unirlas, nace un nuevo esquema de pensamiento, la Toxicología Clínica Comunitaria.

Esimportanteseñalar que si bien la enseñanza de la Toxicología y sobre todo de la Toxicología Clínica todavía es deficiente dentro de la preparación del médico general ${ }^{2}$, existen algunas experiencias en varios países dónde la toxicología clínica ha ido posicionándose en el sector de la salud pública, tales son los casos de Cubaª dónde a través de su programa de Toxicología Comunitaria se lleva el conocimiento de la Toxicología Clínica a la sociedad, o las de Brasi ${ }^{4}$ y M éxi$\mathrm{CO}^{5}$ en su trabajo con sitios contaminados, dondeel seguimiento delas poblaciones expuestas es coordinado por equipos formados con profesionistas de distintas disciplinas provenientes de las Universidades $\mathrm{y} / 0$ de los M inisterios de Salud y Ambiente. También existen experiencias en Argentina, dónde se han creado Unidades Pediátricas Ambientales con un papel destacado de los toxicólogos clínicos médicos y bioquímicos en equipos multidisciplinarios ${ }^{6}$; o las experiencias de evaluaciones en sitios contaminados con plomo, en los que los toxicólogos clínicos argentinos cumplieron un importante rol en la evaluación de los niños expuestos, en las actividades comunitarias de comunicación de riesgos y en la elaboración de medidas de control de exposición?.

Si bien estos ejemplos constituyen un avance en el manejo de poblaciones crónicamente expuestas a sustancias químicas, consideramos que es necesario trabajar en modelos donde haya una clara participación de la Toxicología Clínica en esta problemática. Argentina, Brasil, o Cuba tienen capacidad para involucrar a los toxicólogos clínicos en sus programas de sitios contaminados, dado que cuentan con redes bien establecidas de CIAATs; no obstante, esto no se ha materializado ya que, en muchos casos, los Centros están saturados con el asesoramiento y el manejo de las intoxicaciones agudas.

En conclusión, el trabajo comunitario en escenarios de exposición crónica requiere de todo un equipo multidisciplinario que debeincluir toxicólogos clínicos. No obstante, es importante reconocer queel manejo dela intoxicación ambiental no se agota en el tratamiento al paciente por parte del toxicólogo clínico, pues para lograr el objetivo de reducir el riesgo, se requiere además: dela intervención ambiental, dela solución a problemas estructural es dela comunidad (por ejemplo, sanidad), o el favorecer procesos de educación y el mejorar factores económicos. En este enfoque multidisciplinario, la toxicología clínica debe transformarse para gestar una disciplina mas afín al trabajo en comunidades. La nueva disciplina, que denominamos Toxicología Clínica Comunitaria, precisamente por el contexto multidisciplinario en el que habrá de desenvolverse, se concebirá bajo el concepto de salud colectiva dado queeste concepto es más amplio que el de salud públicå.

Un grupo en toxicología clínica comunitaria requiere profesionistas idóneos para trabajar en los objetivos de la disciplina, a saber: el Peritaje Toxicológico, la Intervención, la Vigilancia y finalmentela Educación (estrategia PIVE). En principio, toda la población debería ser evaluada y atendida, pero es importante hacer notar que, siendo los niños y las mujeres en edad reproductiva los mas susceptibles ${ }^{1}$, deberían ocupar un lugar prioritario.

El peritaje toxicológico consta de cuatro actividades: i) la evaluación ambiental cuyo objetivo es identificar los contaminantes críticos, las rutas de exposición y a partir de datos de contaminación estimar el riesgo empleando metodologías reconocidas9-11; ii) la evaluación toxicológica, tanto de exposición y de efecto a dichos contaminantes (químicos o biológicos) y para lo cual se utilizan, en humanos o en organismos centinelas, biomarcadores de exposición y biomarcadores de efecto biológico; iii) la evaluación clínica que consta de un examen médico con interrogatorio dirigido al riesgo identificado y del levantamiento depruebas clínicas o estudios de gabinete (tipo espirometrías); y iv) el análisis de los factores de vulnerabilidad comunitaria para lo cual se utilizan indicadores sociales, ambientales y sanitarios (incluyendo evaluación por parte de psicólogos comunitarios).

La intervención se refiere a la implementación de las medidas diseñadas para lograr la reducción de los riesgos. Las medidas se dan principalmente en cuatro áreas: i) educación infantil 
en la Casa u hogar, en la Escuela, en los espacios Recreativos y a nivel 0 cupacional en zonas donde aunque no sea lícito, los niños laboren (programa CERO riesgos); ii) medidas ambientales de control de la contaminación; iii) medidas de salud, tales como el mejorar la higiene, la dieta y hasta suministrar al gún antídoto (como en el caso de intoxicaciones crónicas severas por mercurio, plomo, etc); y iv) medidas comunitarias para afectar los factores de vulnerabilidad (calidad de agua, manejo adecuado de basura, programas para el control deadicciones, etc.).

En cuanto a la vigilancia, planteamos un esquema de trabajo que tiene como eje la participación deun CIAAT en el diseño de estudios para el biomonitoreo de químicos y/o en la instrumentación de programas para la evaluación de efectos en salud en los individuos expuestos. Esto último contaría con la participación de personal médico necesariamente capacitado en aspectos toxicológicos. Dos palabras definen la vigilancia: prevención y seguimiento.

Finalmente, la educación médica se refiere ala impartición de cursos o talleres, sobre tópicos diversos en las áreas de toxicología clínica, toxicología ambiental, evaluación de riesgos, etc. Asimismo, se busca la elaboración de guías sencillas de diagnóstico, tratamiento, prevención y vigilancia, cuyo objetivo sería el sensibilizar y capacitar al personal médico que atiende individuos expuestos a sustancias químicas. En este apartado también es importante incluir el diseño de programas de divulgación y de comunicación de riesgos, para que la población en general entienda y atien da los riesgos en salud queenfrenta por la exposición a químicos.

A fin deesquematizar mejor la estrategia PIVE, presentamos datos sobre un caso real. Este caso se presentó en la zona urbana vecina a una Fundición de Arsénico y Plomo, ubicada en la Ciudad de San Luís Potosí, M éxico. Presentamos el caso, explicando las acciones de cada una de las fases, sin embargo, es importante aclarar que en el trabajo cotidiano, las acciones de cada fase muchas veces se dan de manera simultánea ya que, no en raras ocasiones, la intervención debe darse cuando aún no concluyen los estudios sobre evaluación de riesgo.

Caso: metalúrgica dearsénico y plomo

Peritaje toxicológico

La evaluación de riesgos en la zona urbana vecina a la fundición de arsénico y plomo, de- mostró contaminación por ambos metales, sobre todo en aire y suelo12,13; además se estudió la exposición a través de la evaluación del arsénico urinario y del plomo en sangre, encontrándose una alta proporción de niños expuestos con valores por arriba de lo normal12,13. Posteriormente se realizaron trabajos sobre efectos en salud y se demostraron efectos neuropsicológicos asociados a los metales ${ }^{14} \mathrm{y}$ daño pulmonar asociado al bióxido de azufre ${ }^{15}$. Con los datos se apremió a la empresa a mejorar sus sistemas anticontaminantes y sesolicitó la pavimentación del áreaurbana para contener la generación depolvos a partir del suelo contaminado. Asimismo, segestionó el recambio de suelo en las áreas de recreación infantil.

\section{Intervención}

A lo largo de los años se ha trabajado en el área impactada, en aspectos ambientales, educativos y últimamente, clínicos. En aspectos ambientales se logró que la empresa modificara sus procesos y mejorara el equipamiento para el control de emisiones de polvos y gases. Asimismo, la zona urbana se pavimentó y se cambió el suelo en áreas recreativas. En aspectos educacionales se instrumentó un programa que tuvo como audiencia principal, al niño. Este programa consistió en talleres infantiles, obras de teatro, congresos con experimentos educativos, juegos y demás actividades recreativas ${ }^{16}$. El programa se evaluó con base a dibujos elaborados por los niños y se demostró la adquisición de conocimientos para su autoprotección (no comer tierra, lavarse las manos, cortarse las uñas para evitar la acumulación de polvo, limpiar juguetes y vivienda, etc.). Finalmente, en cuanto a la clínica se viene trabajando en la búsqueda de instrumentos deevaluación parael estudio deadolescentes (expuestos durante su infancia a los metales) y de niños para estudiar los efectos de bajos niveles de plomo (ahora que se ha demostrado que este metal es tóxico aún a bajas concentraciones ${ }^{17,18}$.

\section{Vigilancia}

Nuestro grupo ha venido trabajando como CIAAT en el sitio, para la atención de niños expuestos a metales y de niños con problemas respiratorios (este CIAAT se transformará para funcionar como Centro de Toxicología Clínica Comunitaria a partir de enero del 2009). Así, se han diseñado programas de biomonitoreo cada dos años y gracias a ellos hemos demostrado que la exposición infantil a metales se ha reducido deseisa doce veces, a partir dela introducción 
de las medidas de intervención y sin embargo, continuamoscon el programa educativo. Asimismo, recientemente nuestro grupo concluyó un trabajo dónde se cuantificó la exposición a plomo (incluyendo niveles óseos) en adolescentes que estuvieron expuestos a este metal en su infancia (manuscrito en preparación).

\section{Educación médica y divulgación}

Por primera ocasión nuestro grupo impartió cursos de toxicología clínica en el área de estudio (algo no muy fácil dado que M éxico carece de personal suficienteen esta disciplina) y el éxito ha sido tal que ya son tres los cursos impartidos y la Facultad de M edicina de la localidad aceptó difundir el curso como materia optativa de su curriculum para médico cirujano. En cuanto a divulgación, desde el principio, el grupo definió su postura de hacer público los resultados y para ello utilizó tresvías: el diario local; entrevistas personales con los padres de los niños en estudio y asambleas con la sociedad civil. Asimismo, seaceptaron todo tipo de invitaciones para presentar los datos a colegios de profesionistas médicos.

Estecaso, como puedeadvertirsees muy afortunado, pero tenemosya otros ejemplos, en áreas con acuíferos contaminados con flúor ${ }^{19-21}$, en zonas mineras ${ }^{22-24}$, en comunidades indígenas expuestas a humo de leña $a^{25}$ y estamos estudiando varios sitios más.

\section{Comunidad y Clínica}

La Toxicología Clínica Comunitaria implicaría la atención simultánea integral de las intoxicaciones agudas y las crónicas laborales que generalmente se atienden en un ámbito hospitalario y de las intoxicaciones/exposiciones crónicas ambientales que habitualmente se presentan a nivel comunitario (un niño con niveles de plomo en sangreal dobledelo normal difícilmentellega al hospital y en caso de llegar, al no presentar signos de intoxicación aguda, difícilmente tendría el seguimiento requerido para evaluar los efectos neurocognitivos asociados a este metal). Un hecho notable es que en el esquema propuesto, un caso de intoxicación aguda o laboral también puede actuar como "caso índice" para alertar sobre un caso de contaminación y orientar hacia la búsqueda de intoxicaciones ambientales.

El trabajo multidisciplinario permitiría integrar las actividades clínicas con el trabajo comunitario. Por ello, en tanto disciplina, la Toxicología Clínica Comunitaria abarca campos de la toxicología médica, analítica, ambiental, regula- toria y legal, de evaluación de riesgos, así como dela toxicología social y dela comunicación. En tanto desarrollo institucional, un Centro de Toxicología Clínica Comunitaria, debería integrarse con un equipo multidisciplinario, quediera cuenta del ejercicio de los saberes mencionados, y liderado por un Toxicólogo Clínico o un miembro del área dela salud con entrenamiento en el tema y con experiencia en Toxicología Ambiental, lo cual sefundamenta bajo la consideración deque el grupo de toxicología clínica comunitaria atendería casos de intoxicación aguda y crónica.

Es claro que en la mayoría de los países los CIAATs se encuentran con exceso de trabajo y de responsabilidades, con personal limitado y con apoyo económico exiguo. Pero no podemos cerrar los ojos a la realidad de los sitios contaminados 0 al riesgo diario que sufren las comunidades marginadas. Involucrar a los CIAATs en la problemática ambiental y comunitaria podría no ser una medida muy bien recibida por los profesionales que hoy se desempeñan en los CIAATs que se verían sobreexigidos en sus labores, pero si es una acción fundamental para mejorar la salud colectiva de nuestra población mas necesitada. Además, en nuestra experiencia, el involucrarse en temas novedosos permite aumentar la pertinencia de los CIAATs y ello posibilita el acceso a nuevos apoyos económicos. Al final del camino, la Toxicología Clínica Comunitaria podría no solo ser una disciplina que favorece la salud colectiva sino también, un nuevo camino para posibilitar el progreso de la Toxicología Clínica en América Latina.

\section{Colaboradores}

ME Leal, D González, SI García, IN Pérez-M aldonado, E Rico, P Cossío, E Van Brussel, G Domínguez, F Díaz-Barriga participaron igualmente de todas las etapas de elaboración del artículo.

\section{Agradecimientos}

Nuestro agradecimiento al Consejo Nacional de Ciencia y Tecnología de M éxico, Fondo Sectorial Salud (proyecto 13841) y a la Organización Panamericana de la Salud (proyecto GEF/OPS/DDT). 


\section{Referencias}

1. IPCS. Principles for evaluating health risk in children associated with exposure to chemical. Environmental Health Criteria 237; 2006.

2. Suárez-Escandón A. Valoración de la Enseñanza de la Toxicología en los Estudios de M edicina en Emergencias. (Trabajo para optar por el título de Master en Emergencia en Atención Primaria). La Habana: Instituto Superior de Ciencias M édicas. Facultad de Ciencias M édicas "Miguel Enriquez" Centro Nacional de Urgencias M édicas; 2007.

3. Torres-Alemán MA. Las Consultas Comunitarias de Toxicología y su impacto en la calidad de vida en la comunidad. Experiencia en Ciudad de la $\mathrm{Ha}$ bana. Boletín de la Unidad de Espacios SaludablesOPS 2005; 1:2.

4. Fróes-Asmus $C I R$, Palácios $M$, Pessoa da Silva $A$, de Freitas-Filhote MI, Câmara V, Aguilar A, Buosi D. Exposición de niños y adolescentes a residuos peligrosos en Brasil. un estudio de caso. Acta Toxicol Argent 2006; 14(Supl.):20-21.

5. Díaz-Barriga F. Los residuos peligrosos en M éxico. Evaluación del riesgo para la salud. Salud Pública de M éxico 1996; 38(4): 280-291.

6. Unidades Pediátricas en Argentina. (accesado 2008 oct 29). Disponible en: http://www.ambiente.gov. ar/default.asp?! dArticulo $=3419$

7. De Pietri DE, García S, Rico O. Modelos geo-espaciales para la vigilancia local de la salud. Rev $\mathrm{Pa}$ nam Salud Publica 2008; 23(6):394-402.

8. Granda E. ¿A que llamamos salud colectiva, hoy? Rev Cubana de Salud Pública 2004; 30(2). [accesado 2008 oct 29]. Disponible en: http://www.bvs.sld.cu/ revistas/spu/vol30_2_04/spu09204.htm

9. EPA. Environmental Protection Agency. Documentos de Superfund. 2007. [accesado 2008 oct 29]. Disponible en: http://www.epa.gov/superfund/index.htm

10. ATSDR. Public Health Assessment Guidance M anual. Agency for Toxic Substances and Disease Registry. 2008.

11. Díaz-Barriga F. M etodología de Identificación y Evaluación de Riesgos para la Salud en Sitios Contaminados. Organización Panamericana de la Salud, Centro Panamericano de Ingeniería Sanitaria y Ciencias del Ambiente. Perú: Primera Edición, pp 96. OPS/CEPIS/PUB/99.34. World Health Organization. 1999.

12. Díaz-Barriga F, Santos MA, M ejía JJ, Batres L, Yáñez L, Carrizales L, Vera E, Del Razo LM, Cebrian $M E$. Arsenic and cadmium absorption in children living near a smelter complex in San Luis Potosí, M exico. Environ Res 1993; 62(2):242-250.

13. Carrizales L, Razo I, Tellez-Hernandez JI, TorresNerio R, Torres A, Batres LE, Cubillas AC, DiazBarriga $F$. Exposure to arsenic and lead of children living near a copper-smelter in San Luis Potosi, M exico: Importance of soil contamination for exposure of children. Environ Res 2006; 101(1):1-10.

14. Calderón J, Navarro $M E$, Jiménez-Capdeville $M E$, Santos-Díaz M A, Golden A, Rodríguez-Leyva I, Borja-Aburto VH y Díaz-Barriga F. Exposure to arsenic and lead and neuropsychological development in Mexican children. Environ Res 2001; 85(2): 69-76.
15. Leal Pérez ME. Evaluación del riesgo respiratorio en niños expuestos al bióxido de azufre en la Ciudad de San Luis Potosí. [tesis]. San Luis Potosí, SLP: U niversidad Autónoma de San Luis Potosí; 2005.

16. Torres Nerio R. Diseño y aplicación de un programa de comunicación de riesgos para la salud ambiental infantil en un sitio contaminado con plomo y arsénico. [tesis]. San Luis Potosí, SLP: Universidad Autónoma de San Luis Potosí, 2005.

17. García-Vargas GG, Rubio-Andrade M, Del Razo LM, Borja-Aburto, Vera-Aguilar E, Cebrián ME. Lead exposure in children living in a smelter community in region Lagunera, Mexico. J Toxicol Environ Health 2001; 62(6):417-429.

18. Canfield RL, Henderson CR, Cory-Slechta DA, Cox C, Jusko TA, Lanphear BP. Intellectual impairment in children with blood lead concentrations below 10 micrograms per deciliter. N Engl J Med 2003; 348(16):1517-1526

19. Díaz-Barriga F, Navarro-Quezada A, Grijalva M, Grimaldo M, Loyola-Rodríguez JP, Ortíz M D. Endemic Fluorosis in México. Fluoride 1997; 30(4): 233-239.

20. Grimaldo M, Borja V, Ramírez AL, Ponce M, Rosas M, Díaz-Barriga F. Endemic fluorosis in San Luis Potosí, M exico. I. Identification of risk factors associated with human exposure to fluoride. Environ Res 1995; 68(1):25-30.

21. Díaz-Barriga F, Leyva R, Quistián J, Loyola-Rodríguez JP, Pozos A, Grimaldo M. Endemic Fluorosis in San Luis Potosí, M éxico. IV. Sources of Fluoride Exposure. Fluoride 1997; 30(1):219-222.

22. Razo I, Carrizales L, Castro J, Díaz-Barriga $F$ y Monroy M. Arsenic and heavy metal pollution of soil, water and sediments in a semi-arid climate mining area in Mexico. Water Air Soil Pollut 2004; 152(1-4):129-152.

23. Yáñez $L$, García-N ieto $E$, Rojas E, Carrizales $L$, Mejía J, Calderón J, Razol y Díaz- Barriga. DNA damage in blood cells from children exposed to arsenic and lead in a mining area. Environ Res. 2003; 93(3):231-240.

24. Jasso-Pineda Y, Espinosa-Reyes G, Gonzalez-Mille D, Razo-Soto I, Carrizales L, Torres-Dosal A, Mejía-Saavedra J, Monroy M, I rina Ize A, Yarto M, Díaz-Barriga F. An Integrated Health Risk Assessment Approach to the Study of M ining Sites Contaminated With Arsenic and Lead. Integr Environ Assess M anag 2007; 3(3):344-350.

25. Torres Dosal A, Pérez-Maldonado IN, Jasso-Pineda Y, Martínez Salinas RI, Alegría Torres JA, DiazBarriga F. Indoor air pollution in a Mexican indigenous community: Evaluation of risk reduction program using biomarkers of exposure and effect. Sci Total Environ 2008; 390(2-3):362-368.

Artigo apresentado em 13/05/2008

Aprovado em 30/10/2008

Versão final apresentada em 01/12/2008 